\title{
Typing of Aeromonas species by protein fingerprinting: comparison of radiolabelling and silver staining for visualising proteins
}

\author{
SALLY E. MILLERSHIP and SUSAN V. WANT
}

Department of Bacteriology, Royal Postgraduate Medical School, Hammersmith Hospital, Du Cane Road, London W12 ONN

\begin{abstract}
Summary. A method for typing Aeromonas species by silver staining of total soluble proteins separated by sodium dodecyl sulphate-polyacrylamide gel electrophoresis is described. There was good agreement with the results obtained by autoradiography of whole-cell proteins for isolates examined by both methods.
\end{abstract}

\section{Introduction}

Sodium dodecyl sulphate-polyacrylamide gel electrophoresis (SDS-PAGE) of radiolabelled whole-cell proteins has proved useful for typing several bacterial species, including aeromonads (Tabaqchali et al., 1984; Stephenson and Tabaqchali, 1986; Stephenson et al., 1987). However, radiolabelled compounds are expensive and inconvenient to handle, and some bacterial species will not grow readily in the medium required for incorporation of the label. Therefore, silver staining was examined as an alternative means of visualising the proteins. Sensitivity is much better than that of fluorescent stains, heavy metals or Coomassie Brilliant Blue (Patel et al., in press). The procedure is less complex than autoradiography and results are available at least $24 \mathrm{~h}$ earlier.

\section{Materials and methods}

Thirty five isolates of Aeromonas spp., previously typed by autoradiography (Stephenson et al., 1987), were maintained as nutrient-agar stabs with repeated subculture for up to 2 years. They were cultured on horse-blood agar and incubated overnight at $37^{\circ} \mathrm{C}$ in air. Portions of three colonies of each strain were emulsified in $1 \mathrm{ml}$ of phosphate-buffered saline, $\mathrm{pH} 7 \cdot 3$. Sodium dodecyl sulphate (SDS) was added to a final concentration of $2 \%$ $\mathrm{w} / \mathrm{v}$ and the soluble proteins were dissociated by immersing the samples in boiling water for $3 \mathrm{~min}$. The protein concentration was assayed by the method of Lowry et al. (1951) and the samples were diluted with sample buffer (Laemmli, 1970) to $2.5 \mathrm{mg}$ of protein $/ \mathrm{ml}$. SDS-PAGE was performed by the method of Laemmli

Received 25 July 1988; accepted 6 Oct. 1988
(1970) with a stacking gel containing acrylamide $30 \mathrm{~g} / \mathrm{L}$ and a resolving gel containing acrylamide $100 \mathrm{~g} / \mathrm{L}$ at $0.75 \mathrm{~mm}$ thickness. On each gel, 12 samples and molecular weight markers (Bio-Rad high range) were added in $20 \mu \mathrm{l}$ volumes.

Gels were fixed overnight in trichloracetic acid $20 \%$ w/v. Staining was performed as described by Patel et al. (in press). In brief, gels were washed twice with methanol $50 \% \mathrm{v} / \mathrm{v}$ and once with water. They were soaked in silver diamine solution $(21 \mathrm{ml} \mathrm{NaOH} 0.36 \% \mathrm{w} / \mathrm{v}, 1.4 \mathrm{ml} \mathrm{am}-$ monia $35 \% \mathrm{w} / \mathrm{v}, 4 \mathrm{ml}$ silver nitrate $20 \% \mathrm{w} / \mathrm{v}$ ) for $15 \mathrm{~min}$ with constant shaking and washed twice with water. Gels were placed in reducing solution $(2.5 \mathrm{ml}$ citric acid $1 \%$, $0.26 \mathrm{ml}$ formaldehyde $36 \% \mathrm{w} / \mathrm{v}$, made up to $500 \mathrm{ml}$ with water) and proteins were visualised within $10 \mathrm{~min}$. Gels were rinsed in water and stain development stopped by immersion in a solution of methanol $45 \% \mathrm{w} / \mathrm{v}$ containing acetic acid $10 \% \mathrm{w} / \mathrm{v}$.

Wet gels were analysed visually on a light box. The identity of the strains tested was unknown to the investigator who read the profiles. Each pair of protein profiles within a gel was compared according to previously adopted band criteria (Stephenson et al., 1987), and the Dice coefficient (Dice, 1945) was used to calculate similarities between pairs of tracks. The reproducibility of the technique was examined by repeated testing of pairs of prepared extracts of cultures on different gels. Extracts from different colonies of the same strain were also tested in duplicate on the same gel.

The reproducibility of autoradiography was examined by repeated testing of pairs of isolates prepared from the same cultures on each occasion.

\section{Results}

Silver staining of each isolate made possible the recognition of $30-50$ protein bands. The pattern was similar, but not identical to that of autoradiography (fig. 1). 
Fig. 1. Protein profiles obtained after silver staining of whole-cell extracts of Aeromonas spp. separated by SDS-PAGE. Track $1-3, A$. caviae, 1 and 2 are isolates from different members of the same family and 3 is a duplicate preparation of the culture in $2 ; 4-6, A$. hydrophila; 7-8, A. sobria; 9-12, A. caviae; * mol. wt standards of $200,116,93,66$ and 45 . The autoradiographs of isolates in tracks $1,2,3,4,5,7,8,9,10,12$ have been previously published (Stephenson et al., 1987) as track numbers $13,12,12,11,9,8,7,5,4,1$ respectively. The table of similarity coefficients is as follows:

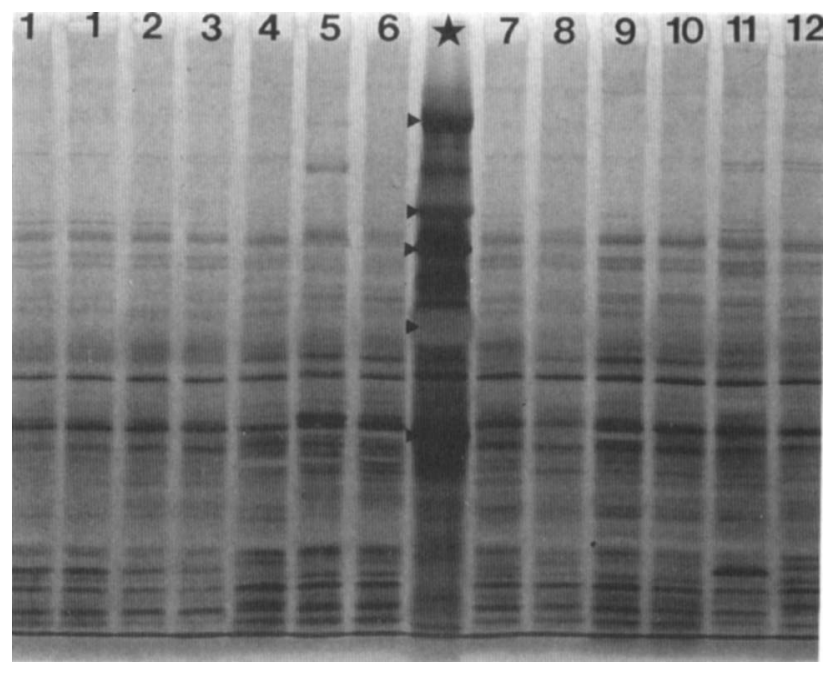

\begin{tabular}{|c|c|c|c|c|c|c|c|c|c|c|c|}
\hline \multirow[b]{2}{*}{ Track no. } & \multicolumn{11}{|c|}{ Similarity coefficient $(\%)$ with Track no. } \\
\hline & 2 & 3 & 4 & 5 & 6 & 7 & 8 & 9 & 10 & 11 & 12 \\
\hline 1 & 90 & 92 & 26 & 16 & 18 & 30 & 30 & 38 & 42 & 70 & 36 \\
\hline 2 & & 96 & 20 & 20 & 24 & 34 & 34 & 54 & 46 & 72 & 34 \\
\hline 3 & & & 40 & 24 & 24 & 42 & 26 & 50 & 38 & 54 & 34 \\
\hline 4 & & & & 28 & 42 & 28 & 44 & 22 & 26 & 26 & 32 \\
\hline 5 & & & & & 74 & 42 & 28 & 34 & 32 & 18 & 20 \\
\hline 6 & & & & & & 22 & 30 & 30 & 28 & 22 & 36 \\
\hline 7 & & & & & & & 32 & 44 & 42 & 62 & 32 \\
\hline 8 & & & & & & & & 48 & 30 & 26 & 30 \\
\hline 9 & & & & & & & & & 68 & 58 & 34 \\
\hline 10 & & & & & & & & & & 52 & 38 \\
\hline 11 & & & & & & & & & & & 40 \\
\hline
\end{tabular}

Similarity coefficients were calculated for 173 pairs of tracks for each method of protein staining. The difference between similarity coefficients was noted for each pair. The mean difference between similarity coefficients was $-9.0 \%$ SD $14.6 \%$, a decrease in similarity on silver staining being taken as negative.

Previously (Stephenson et al., 1987), pairs of isolates more than $80 \%$ similar by autoradiography were considered to be identical, those less than $60 \%$ similar to be different, and those between $60 \%$ and $80 \%$ to be of uncertain relationship. The corresponding criteria for these categories after silver staining were taken as $71 \%$ identical, $51 \%$ different and $51-71 \%$ uncertain, by deduction of the mean difference in similarity between pairs of isolates examined by both methods. The majority $(84.4 \%)$ of pairs were given the same classification (different, indistinguishable or of uncertain relationship) by both methods. Three pairs were indistinguishable by autoradiography but different by silver staining and 24 pairs were of uncertain relationship by one method only (fig. 2). There were 46 pairs in which the strains were of different species; all the similarity coefficients were $<60 \%$ by autoradiography and all except one were $<51 \%$ by silver staining.

Results were also analysed according to the clusters of isolates identified within each species. Where pairs were of uncertain relationship, similarity coefficients of each member of the pair to other isolates were also considered. For example, if $A$ was of uncertain relationship to $C$ but was indistinguishable from $B$ and $B$ from $C$, then $A$ and $\mathrm{C}$ were considered to be indistinguishable. The strains of $A$. hydrophila were divided into two groups of two and six isolates and five single strains by autoradiography. Silver staining divided the largest group into a cluster of three indistinguishable isolates and three of uncertain relationship to the group. Otherwise clustering was identical whichever method was used. Among 18 isolates of A. caviae, five single isolates, three clusters of two, four and five indistinguishable isolates respectively, and a further two isolates of uncertain relationship to the largest cluster were identified. Two isolates 


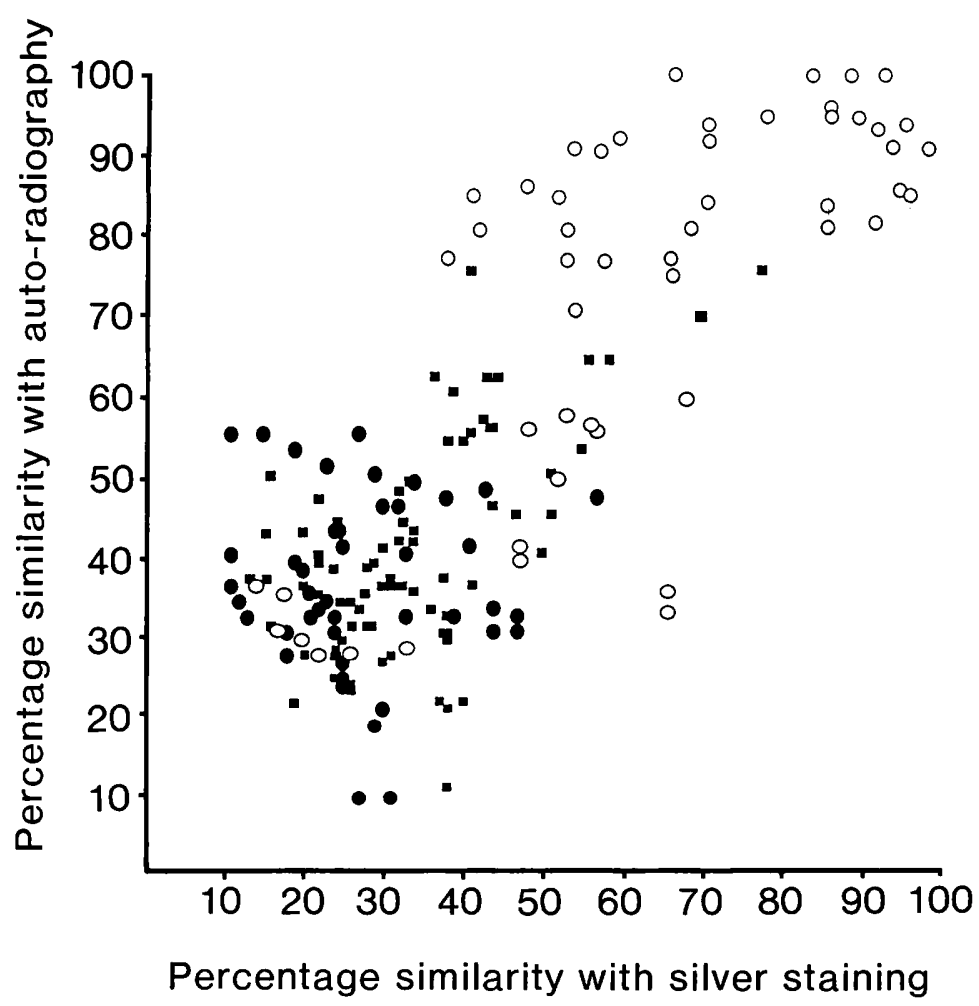

Fig. 2. Comparison of similarity coefficients obtained by silver staining and by autoradiography. Each point represents one pair of strains: pair of isolates from different species; $\square$ pair of isolates from the same species, but epidemiologically unrelated; $\bigcirc$ pair of isolates from the same species, and epidemiologically related.

of $A$. sobria and two not identifiable as any species were not related to any other isolate.

\section{Reproducibility}

Each of 11 pairs of tracks prepared from different colonies of the same strain and stained with silver had $>95 \%$ similarity. The mean difference between the similarity coefficients of 77 pairs of extracts tested on two occasions was $+0.4 \%$ SD $8.9 \%$. A decrease in similarity in a later test was counted as negative. The mean difference between the similarity coefficients of 117 repeated pairs of isolates examined by autoradiography was $6 \cdot 1 \%$ SD $8.0 \%$.

\section{Discussion}

The results of typing by silver staining were in good agreement with those of autoradiography and confirmed the validity of protein fingerprinting as a means of typing. The similarity of pairs of isolates varied little with time or with repeated subcultures.

The reproducibility of typing by silver staining was acceptable in the following respect. Fingerprints of different colonies from one culture compared on the same gel were virtually identical, whereas repeat examination of pairs of stored extracts on different gels showed greater variation. This suggests that the principal source of variability is the running, staining and analysis of the gel which should be amenable to further standardisation, with a corresponding improvement in discrimination. Although further work is required for a strict comparison to be made, it is clear that reproducibility is similar to that of autoradiography.

Pairs of different strains within the same biochemically defined species were as dissimilar as pairs of strains from different species. Aeromonas is known to be heterogeneous with several species as defined by DNA hybridisation within each phenotypic species (Popoff et al., 1981). Distinguishable isolates within a phenotypic species may represent different species by DNA homology. Isolates of uncertain relationship to clusters might represent different strains within a species as defined by DNA homology.

Protein fingerprinting by silver staining represents a useful method of typing, which should be applicable to bacteria of many species.

This work was supported by a Medical Research Council grant. 


\section{REFERENCES}

Dice L R 1945 Measures of the amount of ecological association between species. Ecology 26: 297-302.

Laemmli U K 1970 Cleavage of structural proteins during the assembly of the head of bacteriophage T4. Nature 227: 680685.

Lowry O H, Rosebough N J, Farr A L, Randall R J 1951 Protein measurement with the folin phenol reagent. Journal of Biological Chemistry 193: 265-275.

Patel K, Easty D J, Dunn M J Detection of proteins in polyacrylamide gels using an ultrasensitive silver staining technique. In: Walker $\mathbf{J} \mathbf{M}$ (ed) Methods in molecular biology, vol 2. Humana, Clifton, $\mathrm{NJ}$, in press.
Popoff M Y, Coynault C, Kiredjian M, Lemelin M 1981 Polynucleotide sequence relatedness among motile Aeromonas species. Current Microbiology 5: 109-114.

Stephenson J R, Millership S E, Tabaqchali S 1987 Typing of Aeromonas species by polyacrylamide-gel electrophoresis of radiolabelled cell proteins. Journal of Medical Microbiology 24: 113-118.

Stephenson J R, Tabaqchali S 1986 New method for typing coagulase negative staphylococci. Journal of Clinical Pathology 39: 1271-1275.

Tabaqchali S, Holland D, O'Farrell S, Silman R 1984 Typing scheme for Clostridium difficile: its application in clinical and epidemiological studies. Lancet 1 : 935-938. 